\title{
Grid Resolution Study over Operability Space for a Mach 1.7 Low Boom External Compression Inlet
}

\author{
Bernhard H. Anderson* \\ NASA Glenn Research Center \\ Cleveland, Ohio, 44135
}

\begin{abstract}
This paper presents a statistical methodology whereby the probability limits associated with CFD grid resolution of inlet flow analysis can be determined which provide quantitative information on the distribution of that error over the specified operability range. The objectives of this investigation is to quantify the effects of both random (accuracy) and systemic (biasing) errors associated with grid resolution in the analysis of the Lockheed Martin Company (LMCO) N+2 Low Boom external compression supersonic inlet. The study covers the entire operability space as defined previously by the High Speed Civil Transport (HSCT) High Speed Research (HSR) program goals. The probability limits in terms of a $95.0 \%$ confidence interval on the analysis data were evaluated for four ARP1420 inlet metrics, namely (1) total pressure recovery (PFAIP), (2) radial hub distortion (DPH/P), (3) ) radial tip distortion (DPT/P), and (4) ) circumferential distortion (DPC/P). In general, the resulting $\pm 0.95 \Delta Y$ interval was unacceptably large in comparison to the stated goals of the HSCT program. Therefore, the conclusion was reached that the "standard grid" size was insufficient for this type of analysis. However, in examining the statistical data, it was determined that the CFD analysis results at the outer fringes of the operability space were the determining factor in the measure of statistical uncertainty. Adequate grids are grids that are free of biasing (systemic) errors and exhibit low random (precision) errors in comparison to their operability goals. In order to be $100 \%$ certain that the operability goals have indeed been achieved for each of the inlet metrics, the $Y \pm 0.95 \Delta Y$ limit must fall inside the stated operability goals. For example, if the operability goal for $\mathrm{DPC} / \mathrm{P}$ circumferential distortion is $\leq \mathbf{0 . 0 6}$, then the forecast $\boldsymbol{Y}$ for $\mathrm{DPC} / \mathrm{P}$ plus the $95 \%$ confidence interval on $\mathrm{DPC} / \mathrm{P}$, i.e. $\pm 0.95 \Delta Y$, must all be less than or equal to 0.06 .
\end{abstract}

\section{NOMENCLATURE}

\begin{tabular}{|c|c|}
\hline AIP & Aerodynamic Interface Plane \\
\hline $\mathrm{CCF}$ & Central Composite Face-Centered \\
\hline CFD & Computational Fluid Dynamics \\
\hline $\mathrm{D}_{\mathrm{AIP}}$ & AIP Diameter \\
\hline DES & Detached Eddy Simulation \\
\hline DOE & Design of Experiments \\
\hline $\mathrm{DPH} / \mathrm{P}$ & ARP1420 Radial Hub Distortion \\
\hline $\mathrm{DPC} / \mathrm{P}$ & ARP1420 Circumferential Distortiol \\
\hline
\end{tabular}

* Aerospace Engineer, NASA Glenn Research Center, Cleveland, Ohio, 44135 


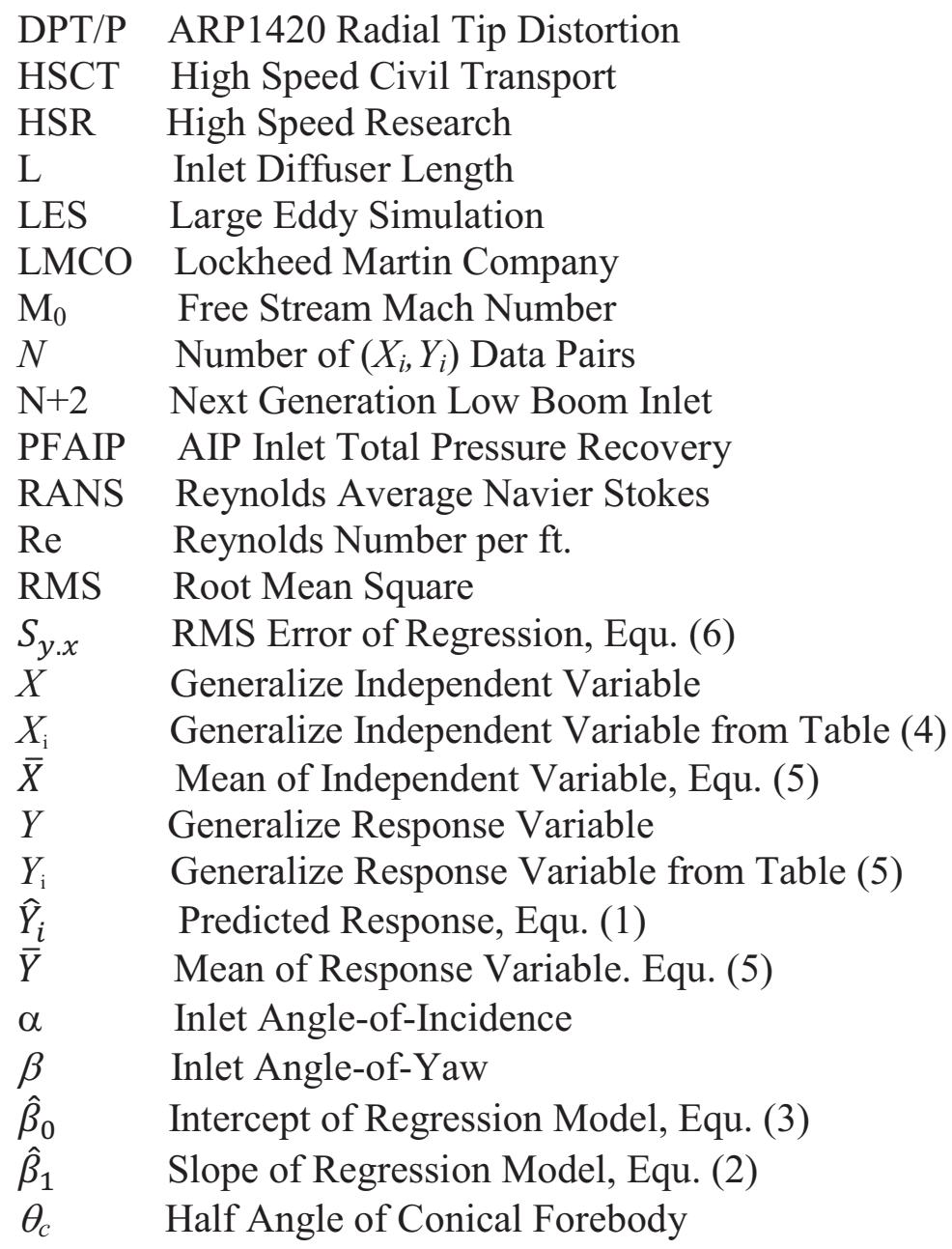

\section{INTRODUCTION}

The National Aeronautics and Space Administration's (NASA) High Speed Project is aligned with the Aeronautics Research Mission Directorate (ARMD) principles of maintaining intellectual stewardship of aeronautics core competencies for the nation in the supersonic flight regime and of focusing research in areas that are appropriate to NASA's unique capabilities. The High Speed Project is a broad based effort designed to develop knowledge, capabilities and technologies that support vehicles that fly in the supersonic speed regime. A major focus of this effort is eliminating the efficiency, environmental and performance barriers to practical supersonic cruise vehicles. The N+2 Supersonic Validation Program focuses on the validation of methodologies to successfully design and develop low boom supersonic air vehicles. As part of this program, the Lockheed Martin Company (LMCO) designed an external compression supersonic low boom inlet which was analytically evaluated at the NASA John Glenn Research Center. Part of the analysis evaluation of the LMCO N+2 inlet was to formalized the issue of grid resolution in terms of statistical methodologies to provide uncertainty limits to the important inlet metrics. 
Scientific journals in almost all disciplines are beginning to require that both measured and computed data contain some indication of statistical uncertainty associated with that data. However, the CFD methods which have been used to test grids do not provide any statistical information regarding the probabilistic limits of the CFD ana;ysis. It also does not provide any quantitate information on the distribution of error over the operability range. For example, a specific grid may provide a small confidence interval at the cruise condition, but fail to provide acceptable probability limits at the outer fringes of the operability space. In addition to calculating the best forecasts of inlet performance over a desired operating range, it is also necessary to specify their accuracy, so that, for example, the risks associated with decisions based upon the forecast may be determined. The measurement of analysis accuracy is also required to objectively (statistically) evaluate whether the analysis either agrees or disagrees with experimental data. While more grid points will always improve the analysis forecast, a computational grid must eventually be specified and the accuracy of that final grid selection evaluated. The accuracy of the forecast may be expressed by calculating the probability limits on either side of the forecast. These limits may be calculated for any convenient probability, for example, $95 \%$. The limits are such that the realized value of the grid effects, when they eventually occur, will be included within these limits and stated probability. For example, for some specified grid selection, we can expect to realize a forecast $Y$ for each of the inlet metrics whose value can vary a $\Delta Y$ amount with $95 \%$ certainty. The goodness of any grid selection then is whether the inlet metric $Y$ is biased or not, and whether one can accept this $\Delta Y$ amount of variation with $95 \%$ certainty for the forecast of that inlet metric. It is therefore the purpose of this study to describe the statistical method used to determine the probability limits $\Delta Y$ of each of the inlet metrics and to examine how these limits vary over operability space.

The objective of this investigation is to quantify the effects of both random (accuracy) and systemic (biasing) errors associated with grid resolution in the analysis of the LMC0 N+2 mixed compression supersonic inlet. The study will cover the entire operability space based on the High Speed Civil Transport (HSCT) ${ }^{(1)}$ High Speed Research (HSR) program goals.

\section{RESULTS AND DISCUSSION}

\section{Inlet Description}

This study will focus on LMCO N+2 Low-Boom Supersonic Inlet Configuration, which will be examined relative to the performance and operability conditions of the HSCT program. The flow path of the LMCO inlet design is schematically shown in Figures (1) and (2). The inlet was designed for a free stream cruise Mach number of 1.70. The centerbody consists of an isentropic compression spike, a curved throat region, and a subsonic diffuser. The compression spike was designed to produce a variable-strength normal shock at the throat with the Mach number ahead of the shock of about 1.25 near the centerbody. The shock was weak at the centerbody to minimize shock/boundary-layer interaction and was stronger at the cowl lip. The forward centerbody of this inlet had a conical half angle, $\theta_{c}$ of 10.0 degrees, while the subsonic diffuser was very short with an $\mathrm{L} / \mathrm{D}_{\mathrm{AIP}}$ of 1.116 . Operability space was based on the goals for HSCT, Figure (4). The operability goals for the HSCT program were originally specified for a Mach 2.5 mixed compression inlet. Since the present inlet is a Mach 1.7 external compression 
inlet, the recovery goal was ignored, while the remaining operability goals were considered desirable for the LMCO N+2 inlet as a basis for judgment. The factor variables and max-min values for the HSCT operability goals are the free stream Mach number $\mathrm{M}_{0}(1.60-1.80)$, the angle-o-attack $\alpha\left(0.0^{\circ}-4.0^{\circ}\right)$ and the angle-of-yaw $\beta\left(0.0^{\circ}-4.0^{\circ}\right)$.

\section{CFD Analysis Method}

There was no information provided for the inlet definition downstream of the AIP station. Therefore, to complete the inlet grid, a constant area section was constructed with a length ratio $\Delta \mathrm{L} / \mathrm{D}_{\text {AIP }}$ of 1.0 followed by a convergent-divergent nozzle to control the inlet mass flow. Table (1) summarizes the two computational grids that were constructed for this study. A "standard grid" composed of $3.461 \times 10^{6}$ grid points and a "fine grid" containing $27.686 \times 10^{6}$ grid points. The "fine grid" was obtained by doubling the grid dimension of the "standard grid" in each of the X, Y, Z directions. The grid topology for this inlet is shown in Figure (3). There were a total of 66 blocks in each of the solution grids. Each of the RANS cases reported herein paper were run with the Wind-US V3.0 Reynolds-averaged Navier-Stokes code ${ }^{(2)}$.

\section{Design of the Grid Resolution Evaluation}

In order to generate a wide range of inlet operating conditions to evaluate the standard grid, a Design of Experiment (DOE) approach $^{(3)}$ was used to span the operability space. Table (2) shows the operational variables held constant throughout the statistical study, while Table (3) shows the factor variables and the ranges studied. The factor variables included the free stream Mach number $\left(\mathrm{M}_{0}\right)$, angle-of attack, $(\alpha)$, and angle-o-yaw, $(\beta)$. The response variables in the DOE layout are shown in Table (4) and were the standard US ARP1420 ${ }^{(4)}$ total pressure recovery (PFAIP), the circumferential distortion descriptor (DPC/P), the radial hub distortion descriptor (DPH/P), and radial tip distortion descriptor (DPT/P), all at the critical inlet operating condition For the analysis in this paper, $Y$ is the measured metric from the standard grid solution (Table 5) and $X$ is the reference or true metric value from the fine grid analysis (Table 6). The sample statistical population was defined by a three variable, three level Central Composite Face Centered (CCF) Design-of-Experiment (DOE) plus two additional cases for lack of fit evaluation. This DOE design is shown graphically in Figure (5). The performance values for the standard grid DOE are shown in Table (5), while the fine grid performance results are presented as a DOE in Table (6). A statistical population of 17 samples was thus defined for both grids, which were a matched pair and are statistically significant and completely spanned the HSCT operability space. The various kinds of biasing (systemic) errors and random (precision) that occurred due to grid resolution were then determined using a statistical methodology described by Anderson and Keller ${ }^{(5)}$. 


\section{Statistical Analysis of the CFD Data}

In order to understand the various kinds of random (precision) and systematic (biasing) errors that were introduced into the estimation of the total pressure and distortion levels as a result of grid resolution, an ordinary least square regression (OLR) was employed for analyzing the relationship between the standard grid results and the reference or fine grid values.

Comparison of the Least Squares Regression line with the ideal functional relationship (i.e. oneto-one line of perfect agreement) provided important information as to the nature of error, either random (precision) or systematic (bias). The method of least squares defines the "best fitting" model to be the one that comes closest to the sample data in the sense of minimizing the sum of squared discrepancies between the observed response value and the respective value predicted by the regression model. A simple linear regression model is given by the expression:

$$
Y_{i}=\beta_{0}+\beta_{1} X_{i}
$$

The slope $\beta_{1}$ and intercept $\beta_{0}$ of equation (1) are estimated by:

$$
\hat{\beta}_{1}=\frac{\sum_{i=1}^{N}\left(X_{i}-\bar{X}\right)\left(Y_{i}-\bar{Y}\right)}{\sum_{i=1}^{N}\left(X_{i}-\bar{X}\right)^{2}}
$$

and

$$
\hat{\beta}_{0}=\bar{Y}-\hat{\beta}_{1} \bar{X}
$$

Where $\bar{X}$ is the mean of the independent or reference variable.

$$
\bar{X}=\frac{1}{N} \sum_{i=1}^{N} X_{i}
$$

and $\bar{Y}$ is the mean of the dependent or response variable:

$$
\bar{Y}=\frac{1}{N} \sum_{i=1}^{N} Y_{i}
$$

and $N$ is the number of $\left(X_{i}, Y_{i}\right)$ pairs of data samples. The RMS error $S_{y . x}$ of the regression is given by:

$$
S_{y \cdot x}=\sqrt{\frac{\sum_{i=1}^{N}\left(Y_{i}-\bar{Y}\right)^{2}}{N-2}}
$$

With $95.0 \%$ confidence, the actual or true line falls in the region bounded by the hyperbolic curves described by: 


$$
\pm 0.95 Y=\hat{Y} \pm t(0.975, N-2) S_{y \cdot x} \sqrt{\frac{1}{N}+\frac{(X-\bar{X})^{2}}{\sum_{i=1}^{N}\left(X_{i}-\bar{X}\right)^{2}}}
$$

and with $95.0 \%$ confidence, the analysis data will be bounded within the region defined by the expression:

$$
\pm 0.95 Y=\hat{Y} \pm t(0.975, N-2) S_{y \cdot x} \sqrt{1+\frac{1}{N}+\frac{(X-\bar{X})^{2}}{\sum_{i=1}^{N}\left(X_{i}-\bar{X}\right)^{2}}}
$$

where $\hat{Y}$ is the predicted response value using Equ. (1), $t(0.975, N-2)$ is the critical value from the t-tables, and $\bar{X}$ is the mean of the independent or reference metric variable. Likewise, $95.0 \%$ confidence intervals can be placed on both the slope of the regression model, $\pm 0.95 \beta_{1}$ and the intercept of the regression model, $\pm 0.95 \beta_{0}$ from the expressions:

$$
\begin{gathered}
\pm 0.95 \beta_{1}=\hat{\beta}_{1} \pm t(0.975, N-2) \frac{S_{y \cdot x}}{\sqrt{S_{x}^{2}(N-2)}} \\
\pm 0.95 \beta_{0}=\hat{\beta}_{0} \pm t(0.975, N-2) S_{y \cdot x} \sqrt{\frac{1}{N}+\frac{\bar{X}^{2}}{S_{x}^{2}(N-1)}}
\end{gathered}
$$

Where $S_{x}{ }^{2}$ is given by the expression:

$$
S_{x}{ }^{2}=\frac{\sum_{i=1}^{N}\left(X_{i}-\bar{X}\right)^{2}}{(N-1)}
$$

\section{Statistical Impact of Grid Resolution}

The performances of the "standard grid" 3D RANS analysis results as measured by the ARP1420 inlet metrics of total pressure recovery (PFAIP), radial hub distortion (DPH/P), radial tip distortion (DPT/P), and circumferential distortion (DPC/P) are presented in Figures (6) through (8). Each figure contains two "scatter" graphs. In the a-series, the $95 \%$ confidence interval is on the "true line" as determined by Equ. (7), while in the b-series of figures, the $95 \%$ confidence interval is for the "analysis data" computed from Equ. (8). Presented in each figure is the one-to-one line of perfect agreement (ideal relationship) between the fine grid reference metric and the standard grid measured metric. The various kinds of systemic (biasing) errors that occurred between the fine grid and standard grid solutions can be seen by comparing the least square regression line with the ideal functional relationship for each of the inlet metrics (Yi) presented in the a-series of graphs. If the one-to-one line of perfect agreement (ideal line) falls completely within the $95 \%$ confidence interval on the "true line", then there is no bias (i.e. regression line is not statistically significantly different from the line of perfect agreement). To augment these visual summaries, the results of the calculating the $95 \%$ confidence intervals on 
the slopes and intercept are presented in Table (7). These are also useful in assessing measurement biasing. If the $95 \%$ confidence interval on the slope contains a slope of 1.0 and the $95 \%$ confidence interval on the intercept contains the intercept 0 , then the regression line is not statistically different from the ideal or one-to-one line of perfect agreement. For example, the $\pm 95 \%$ confidence interval on the slope of DPC/P is 0.77289 and 1.11753 , Table (7). Since the slope of the ideal line is 1.0 and falls within 0.77289 and 1.11753 , regression line is not statistically different from the ideal or one-to-one line of perfect agreement. However, confidence intervals on the intercept that are far removed from the origin (i.e. $(X, Y)=0.0)$ can be unusually large and therefore meaningless. Hence testing the intercept for PFAIP in this study was not applicable (i.e. N/A). Testing for bias by testing on the slope of PFAVE for being significantly different from one and testing whether the one-to-one line of perfect agreement falls within the $95 \%$ confidence interval of the true regression line are both valid and meaningful.

For example, in Figure (6a) the one-to-one line of perfect agreement almost falls within the confidence interval on the true line for the PFAIP total pressure recovery. However, since the $95 \%$ PFAIP confidence interval on the slope $\pm 0.95 \beta_{0}$ contains the number one in Table (7), the regression line is not judged to be statistically different from the line of perfect agreement. In other words no biasing takes place in calculating PFAIP using the standard grid. However, biasing takes place in the calculation of DPH/P and DPT/P using the standard grid and this can be seen if Figures (7a) and (8a). This biasing can also be seen in Table (7) because the $95 \%$ confidence interval on the slope $\pm 0.95 \beta_{0}$ for these two metrics does not contain the number one. Finally, no statistical biasing took place in computing the circumferential distortion DPC/P using the standard grid as indicated in Figure (9a), and this is verified in Table (7). Notice, that these findings are easier to see visually in the Figures (6) through (9) that show the 95\% confidence interval on the true line, but are substantiated in the Table (7) which provide the 95\% confidence on the slopes $\pm 0.95 \beta_{1}$ and intercepts $\pm 0.95 \beta_{0}$ of the regression model

The goodness of any grid selection then is whether there is or there is not any biasing in the calculation of inlet responses $Y$ and that one can accept this $\Delta Y$ amount of variation with $95 \%$ certainty for the forecast of the inlet metric $Y$. This variation is termed the probability limits and is characterized by the $\pm 0.95 \Delta Y$ interval determined from the equation (8). These limits may be calculated for any convenient probability, but $95 \%$ was chosen because it is an industry standard. The probability limits for the for ARP1420 inlet metrics in this study, i.e. AIP total pressure recovery (PFAIP), radial hub distortion, radial tip distortion (DPT/P), and circumferential distortion (DPC/P) are presented in the b-series of scatter plots in Figures (6) through (8). In the b-series of scatter plots, it is the $\pm 0.95 \Delta Y$ interval on the analysis data determined by equation (8) that is being presented. The probability limits for the inlet metrics in this study as indicated by the $\pm 0.95 \Delta Y$ interval are summarized in Table (9) and compared to the HSCT operability goals. The $\pm 0.95 \Delta Y$ interval presented in Table (9) is the average over operability space, where operability space is defined by the HSCT goals. Figure (10) presents the $\pm 0.95 \Delta Y$ probability limits over operability space shown as the $(\alpha * \beta)$ interaction at $\mathrm{M}_{0}=1.7$ response surface for each of the inlet metrics. Once established, $\pm 0.95 \Delta Y$ probability limits do not appreciably change over operability space, i.e. they are essentially constant. Thus, the $\pm 0.95 \Delta Y$ probability limits for the AIP total pressure recovery (PFAIP) is \pm 0.01072 , and the $\pm 0.95 \Delta Y$ probability limits for the ARP1420 radial hub distortion (DPH/P) is \pm 0.01011 , radial tip distortion (DPT/P) is \pm 0.02051 , 
and circumferential distortion (DPC/P) is \pm 0.02066 . The significance of these probability limits becomes clear when compared to the HSCT nominal distortion goals shown in Table (9). Thus the error in computing the DPH/P distortion is about 2/3 the HSCT goal, while the error in computing the DPT/P distortion is actually greater than the nominal HSCT goal, and the error in computing the DPC/P distortion is also about $2 / 3$ of the HSCT goal. In order to be $100 \%$ certain that the operability goals have indeed been achieved for each of the inlet metrics, the $Y \pm 0.95 \Delta Y$ must be less than or equal to the stated operability goals.

To understand the mechanism that is driving the large $\pm 0.95 \Delta Y$ probability limits for the standard grid analysis results, it is best to examine Figure (6b), which presents the PFAIP error characteristics over the HSCT operability space. The large $\pm 0.95 \Delta Y$ standard deviation resulted from the large errors encountered in computing PFAVE at the outer fringes of operability space, i.e. the five analysis points in the lower left corner of Figure (6a). Without these analysis points, the standard grid results would exhibit very small random errors. These error characteristics can also be seen in Figures (7) through (9). It is the error characteristics on the outer fringes of operability space, (i.e., in regions of higher Mach numbers, angles-of-attack and high angles-ofyaw) that dictate the choice of an adequate grid. Adequate grids are grids that are free of biasing (systemic) errors and exhibit low random (precision) errors in comparison to their operability goals.

One bit of explanation is warranted regarding these confidence intervals. The amount of scatter in the data also affects the results. The "noisier" the data (i.e. the larger the standard deviation of the regression, $(S y . x)$ the larger the confidence interval becomes. Larger confidence intervals tend to lead to the conclusion that there is no bias. While this may be statistically true, it is a poor trade-off for having larger random measurement errors. There may be no detectable bias, but the random measurement variation is poor. An example of this poor trade-off is the statistical results for evaluation PFAIP in Figure (6) and DPC/P in Figure (9). The opposite is also true. If there is very little scatter in the data (i.e. about the fitted regression line), the $S y . x$ is very small and the $95 \%$ confidence intervals become extremely tight leading to the conclusion of a significant, detectable bias. So one must view the data and statistical results with a properly focused eye taking into account the amount of random error in the data, and thus the standard warning of statistical versus practical differences.

\section{SUMMARY OF RESULTS}

Scientific journals in almost all disciplines are beginning to require that tables on results contain some measure of statistical uncertainty associated with the data presented, whether that data was measured or computed. This paper presents a statistical methodology whereby the probability limits associated with CFD grid resolution of inlet flow analysis can be determined and which provides quantitative information on the distribution of that error over the specified operability range. The objective of this investigation is to quantify the effects of both random (accuracy) and systemic (biasing) errors associated with grid resolution in the analysis of the LMC0 N+2 Low Boom mixed compression supersonic inlet. The study covers the entire operability space as defined by the High Speed Civil Transport (HSCT) High Speed Research (HSR) program goals. Two computational grids were constructed for this study. A "standard 
grid" composed of $3.461 \times 10^{6}$ grid points and "fine grid" containing $27.686 \times 10^{6}$ grid points. The "fine grid" was obtained by doubling the grid dimension of the "standard grid" in each of the X, $\mathrm{Y}$, and $\mathrm{Z}$ directions. There were a total of 66 blocks in each of the solution grids and the WindUS V2.0 Reynolds-averaged Navier-Stokes code was used in the inlet flow analysis.

The probability limits in terms of a $95.0 \%$ confidence on the analysis data was evaluated for the four ARP1420 inlet metrics, namely (1) total pressure recovery (PFAIP), (2) radial hub distortion, (3) radial tip distortion (DPT/P), and (4) circumferential distortion (DPC/P), over operability space specified by the HSCT program. In general, the resulting $\pm 0.95 \Delta Y$ was unacceptably large in comparison to the stated goals of the HSCT program. Therefore, the conclusion was reached that the "standard grid" size was insufficient for this type of analysis. However, in examining the statistical data, it was determined that the CFD analysis results at the outer fringes of operability space were the determining factor in the measure of statistical uncertainty. Adequate grids are grids that are free of biasing (systemic) errors and exhibit low random (precision) errors in comparison to their operability goals. In order to be $100 \%$ certain that the operability goals have indeed been achieved for each of the inlet metrics, the $Y \pm 0.95 \Delta Y$ must be less than or equal to the stated operability goals.

The goodness of any grid selection then is whether the inlet metric $Y$ is biased or not, and whether one can accept this $\Delta Y$ amount of variation with $95 \%$ certainty for the forecast of that inlet metric. While this study did not provide specific statistical inferences about the "goodness" of the "fine" grid, which was a factor of eight times the "standard" grid, we can infer that the fine grid results will have much smaller probability limits than the standard grid results, particularly at high angles-of-attack and angles-of-yaw, and thus more acceptable for practical use.

\section{REFERENCES}

1. Vietch, L. C., and Hong, W. S., "The National Aeronautics and Space Administration High Speed Research Program and DoD Propulsion Technology Interchange Workshop", Institute Defense Analysis (IDA) Report D-2354, 1999.

2. Towne, C.E. “Wind-Us User's Guide, Version 2.0, NASA TM-2009-215804, 2009.

3. Box, E. P., Jenkins, G. M., and Reinsel, G. C.: “Time Series Analysis”, John Wiley \& Sons, 2008

4. Society of Automotive Engineers, Inc., "Gas Turbine Engine Inlet Flow Distortion Guidelines," ARP1420 Rev B, 2002.

5. Anderson, B.H. and Keller, D. J., "Considerations in the Measurement of Inlet Distortion for High Cycle Fatigue in Compact Inlet Diffusers”, NASA TM 2002-211476, 2002.

American Institute of Aeronautics and Astronautics 


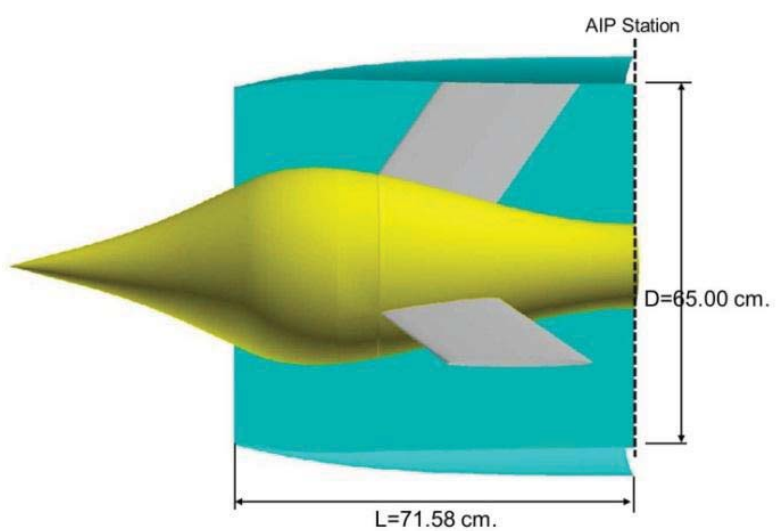

Figure (1): Schematic diagram of $L M C O N+2$ inlet design, $L / D_{\text {aip }}=1.116$

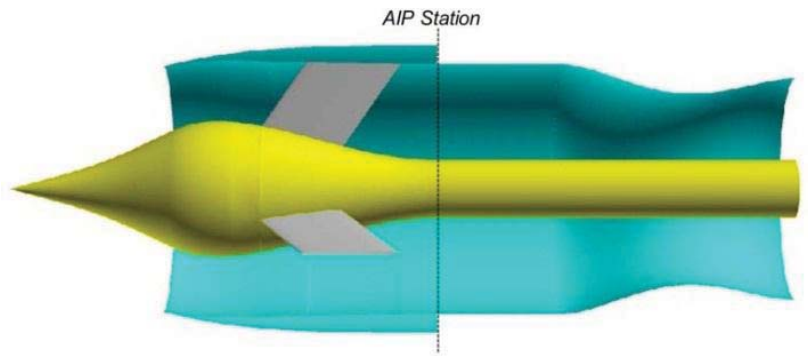

Figure (2): Schematic diagram of LMCO N+2 inlet design.

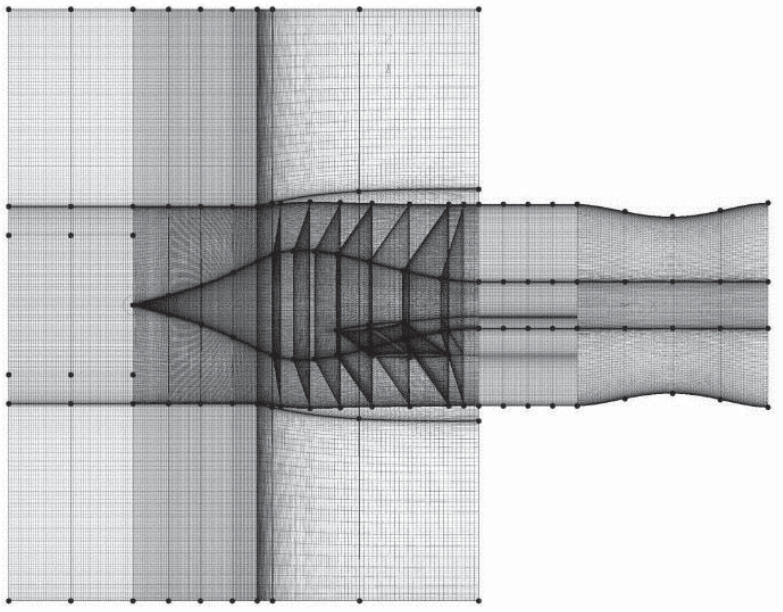

Figure (3): Computational grid for LMCO N+2 inlet design, Grid $=31.482 \times 10^{6}$, Blocks $=66$.

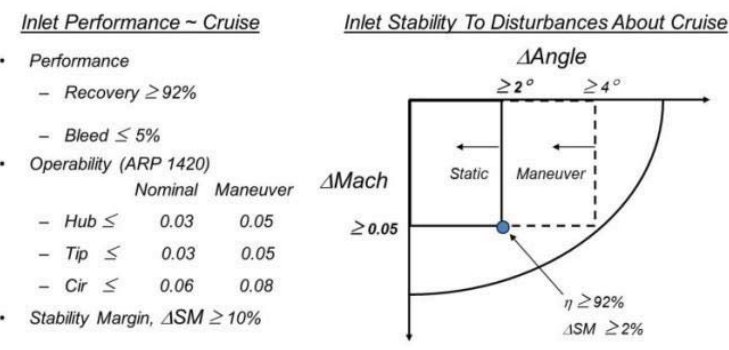

Figure (4): High Speed Civil Transport (HSCT) High Speed Research (HSR) operability goals.

\begin{tabular}{|c|c|}
\hline Grid & Grid Size \\
\hline Standard Mesh & $3.461 \times 10^{6}$ \\
\hline Fine Mesh & $27.686 \times 10^{6}$ \\
\hline
\end{tabular}

Table (1): Comparison of standard and fine mesh grids.

\begin{tabular}{|c|c|}
\hline Variable & Value \\
\hline Tunnel Total Pressure $\left(\mathrm{Ibs} / \mathrm{ft}^{2}\right), \mathrm{P}_{\circ}$ & 2112.0 \\
\hline Tunnel Total Temperature $\left({ }^{\circ} \mathrm{R}\right), \mathrm{T}_{\circ}$ & 512.0 \\
\hline
\end{tabular}

Table (2): DOE variables held constant.

\begin{tabular}{|c|c|}
\hline Response Variable & Symbol \\
\hline AIP Critical Total Pressure Recovery & PFAIP \\
\hline AIP Circumferential Distortion & DPC/P \\
\hline AIP Radial Hub Distortion & DPH/P \\
\hline AIP Face Radial Tip Distortion & DPT/P \\
\hline
\end{tabular}

Table (4): DOE ARP1420 response variables.

\begin{tabular}{|c|c|}
\hline Factor Variable & Range \\
\hline Free Stream Mach Number, $\mathrm{M}_{0}$ & $1.6-1.8$ \\
\hline Free Stream Angle of Attack, $\alpha$ & $0.0^{\circ}-4.0^{\circ}$ \\
\hline Free Stream Angle of Yaw, $\beta$ & $0.0^{\circ}-4.0^{\circ}$ \\
\hline
\end{tabular}

Table (3): DOE factor variables and range.

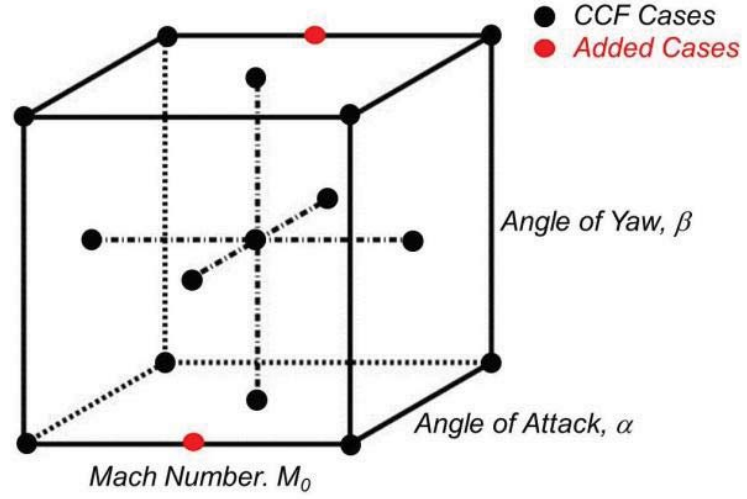

Figure (5): Schematic diagram of DOE operability design.

\begin{tabular}{|l|l|l|l|l|l|l|l|}
\hline Case & $M_{0}$ & $\alpha$ & $\beta$ & PFAIP & DPH/P & DPT/P & DPC/P \\
\hline NPT700 & 1.70 & 0.0 & 0.0 & 0.96291 & 0.07622 & 0.04439 & 0.07288 \\
\hline NPT701 & 1.60 & 0.0 & 0.0 & 0.97944 & 0.06382 & 0.02455 & 0.05684 \\
\hline NPT702 & 1.80 & 0.0 & 0.0 & 0.91596 & 0.04226 & 0.12683 & 0.09265 \\
\hline NPT703 & 1.60 & 4.0 & 0.0 & 0.97490 & 0.05984 & 0.02696 & 0.05579 \\
\hline NPT704 & 1.80 & 4.0 & 0.0 & 0.90259 & 0.04884 & 0.11551 & 0.07893 \\
\hline NPT705 & 1.60 & 0.0 & 4.0 & 0.97749 & 0.05362 & 0.02882 & 0.09782 \\
\hline NPT706 & 1.80 & 0.0 & 4.0 & 0.91378 & 0.04739 & 0.12796 & 0.13982 \\
\hline NPT707 & 1.60 & 4.0 & 4.0 & 0.96123 & 0.07329 & 0.01547 & 0.09685 \\
\hline NPT708 & 1.80 & 4.0 & 4.0 & 0.90548 & 0.04113 & 0.11819 & 0.11933 \\
\hline NPT709 & 1.60 & 2.0 & 2.0 & 0.97875 & 0.06238 & 0.02407 & 0.09610 \\
\hline NPT710 & 1.80 & 2.0 & 20 & 0.91375 & 0.04914 & 0.12886 & 0.12820 \\
\hline NPT711 & 1.70 & 0.0 & 2.0 & 0.96417 & 0.06108 & 0.04275 & 0.11459 \\
\hline NPT712 & 1.70 & 4.0 & 2.0 & 0.94945 & 0.07541 & 0.07484 & 0.10804 \\
\hline NPT713 & 1.70 & 2.0 & 0.0 & 0.96917 & 0.06729 & 0.04791 & 0.06917 \\
\hline NPT714 & 1.70 & 2.0 & 4.0 & 0.95727 & 0.06113 & 0.05321 & 0.11960 \\
\hline NPT715 & 1.70 & 2.0 & 2.0 & 0.95970 & 0.06206 & 0.05097 & 0.11418 \\
\hline NPT716 & 1.70 & 4.0 & 4.0 & 0.94246 & 0.07455 & 0.05253 & 0.11611 \\
\hline
\end{tabular}

Table (5): Central composite face centered DOE operability design, performance results, standard grid solutions, Grid $=3.461 \times 10^{6}$

American Institute of Aeronautics and Astronautics 


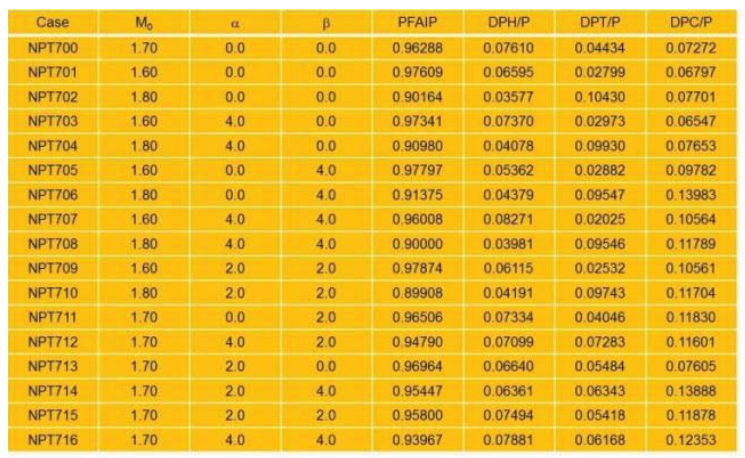

Table (6): Central composite face centered DOE operability design, performance results, fine grid solutions, Grid $=27.686 \times 10^{6}$

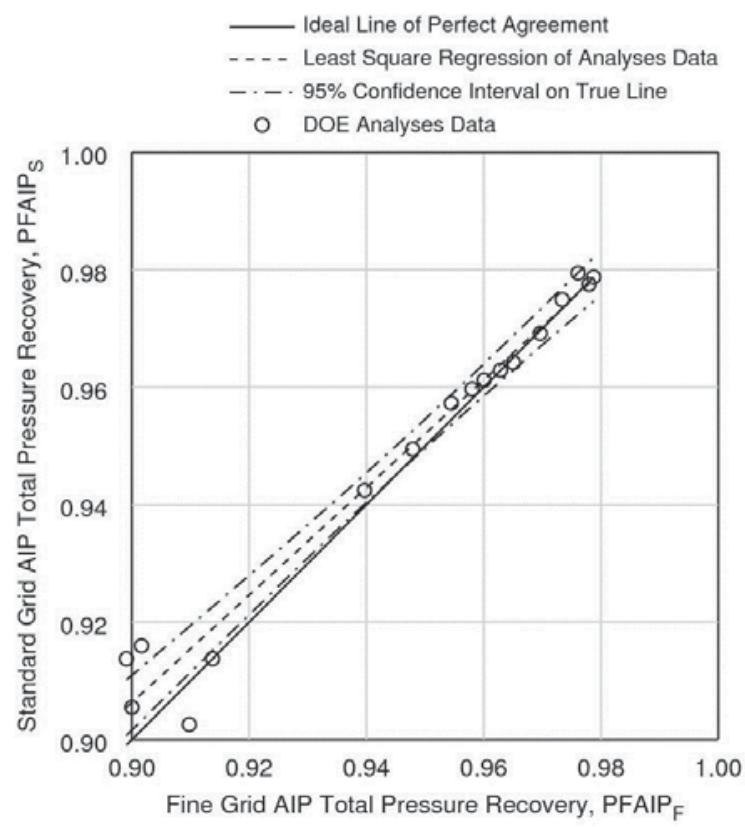

(a) $95 \%$ Confidence interval on true line

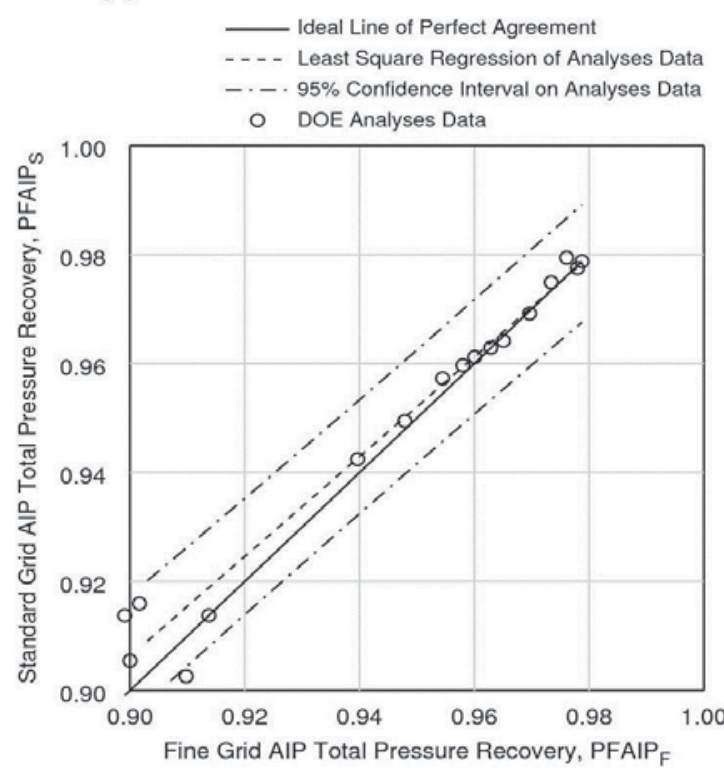

(b) $95 \%$ Confidence interval on analysis data

Figure (6): Total pressure recovery (PFAIP) statistical error characteristics.

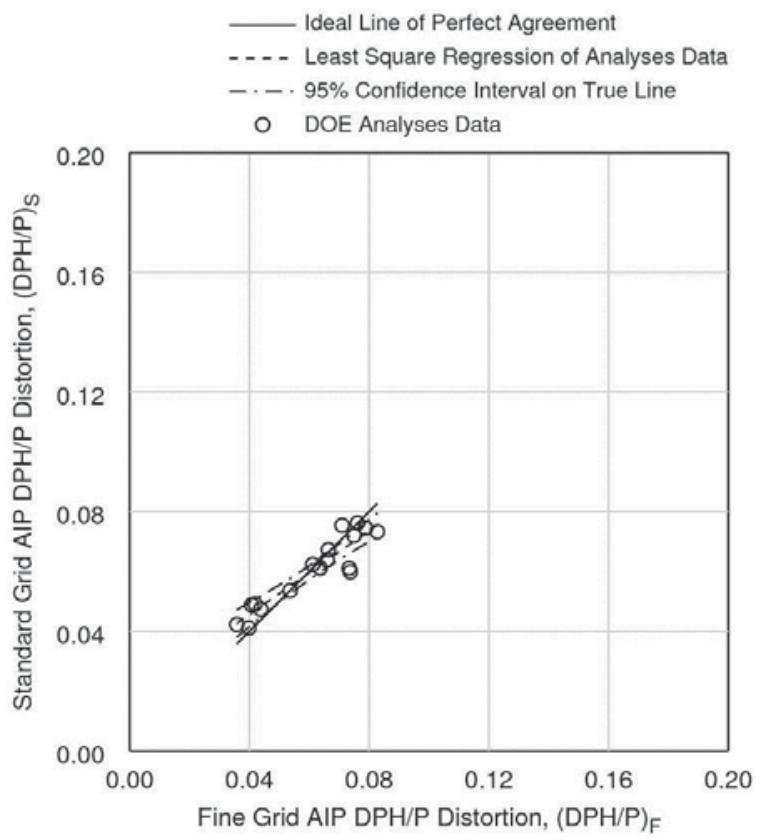

(a) $95 \%$ Confidence interval on true line

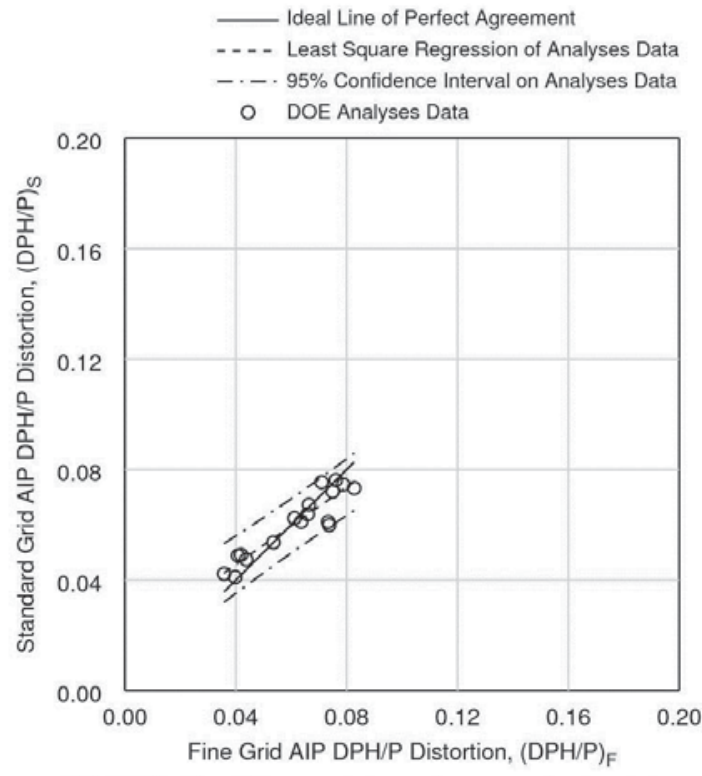

(b) $95 \%$ Confidence interval on analysis data

Figure (7): ARP 1420 radial hub distortion $\mathrm{DPH} / \mathrm{P}$ statistical error characteristics.

American Institute of Aeronautics and Astronautics 


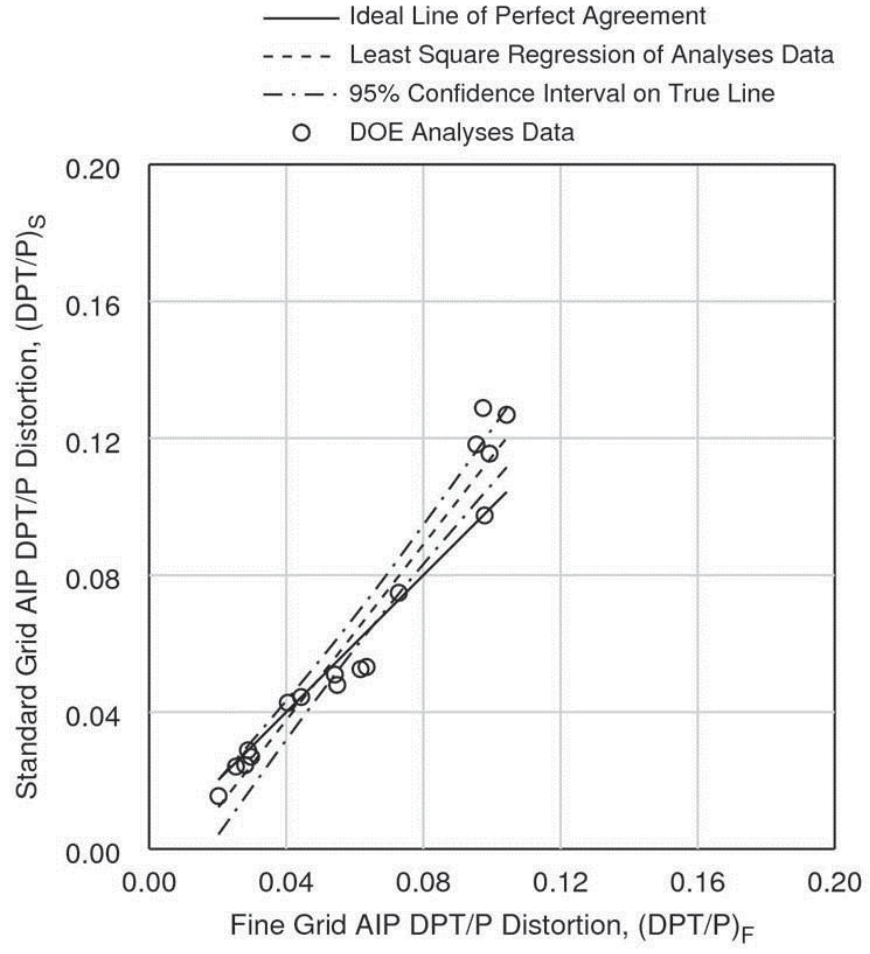

(a) $95 \%$ Confidence interval on true line.

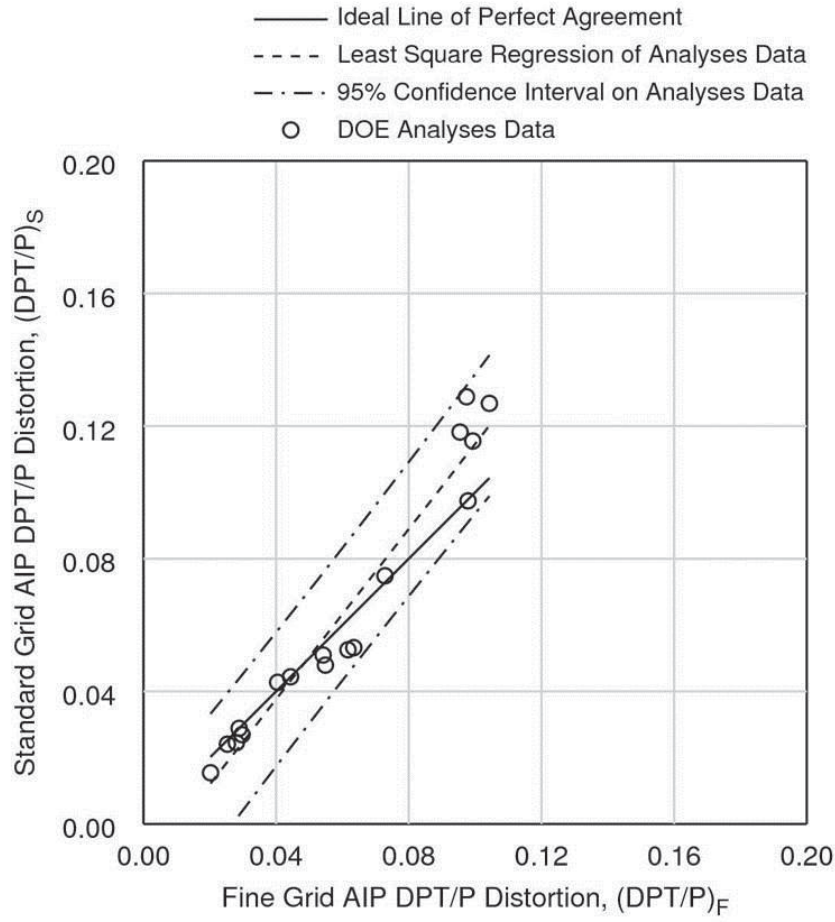

(b) $95 \%$ Confidence interval analysis data

Figure (8): ARP 1420 radial tip distortion DPT/P statistical error characteristics.
_ Ideal Line of Perfect Agreement

- - - - Least Square Regression of Analyses Data

-. - . 95\% Confidence Interval on True Line

O DOE Analyses Data

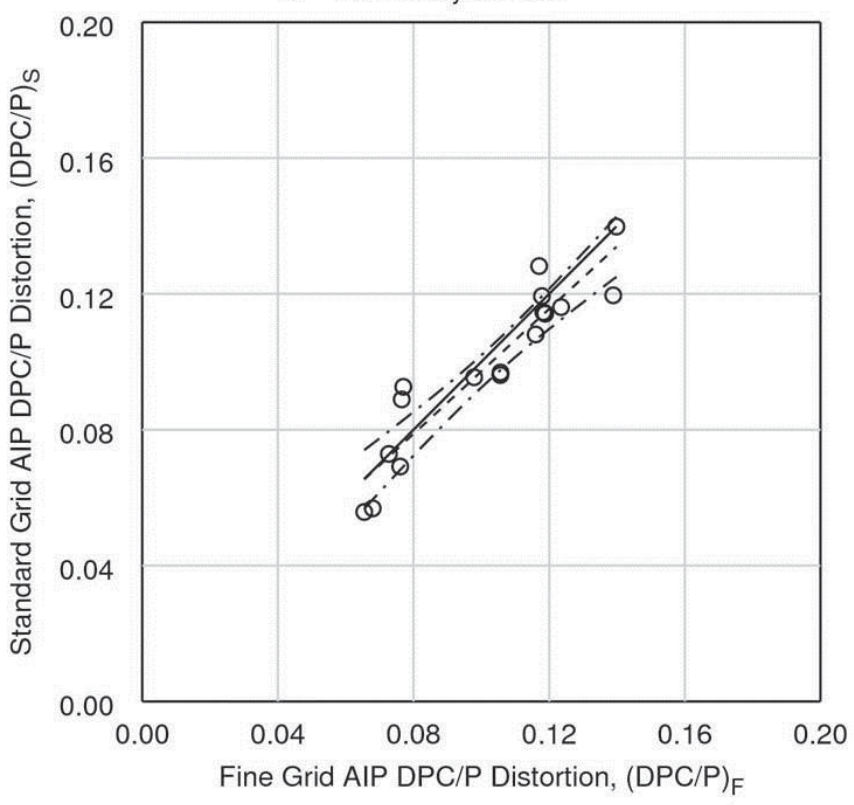

(a) $95 \%$ Confidence interval on true line.

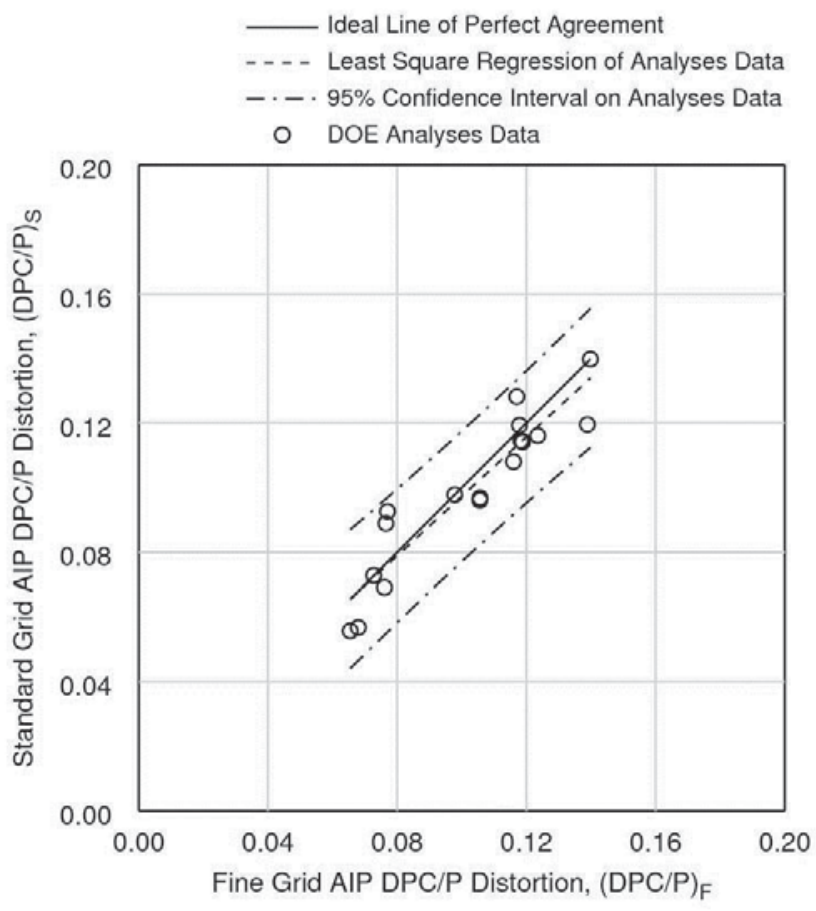

(b) $95 \%$ Confidence interval on analysis data

Figure (9): ARP 1420 circumferential distortion DPT/P statistical error characteristics.

American Institute of Aeronautics and Astronautics 


\begin{tabular}{|c|c|c|c|c|}
\hline Coeff. & PFAIP & DPH/P & DPT/P & DPC/P \\
\hline$-0.95 \beta_{0}$ & 0.00122 & -0.02352 & -0.02351 & -0.01497 \\
\hline$\beta_{0}$ & 0.08222 & -0.01376 & -0.01375 & 0.00517 \\
\hline$+0.95 \beta_{0}$ & 0.16322 & -0.00399 & -0.00399 & 0.02531 \\
\hline$-0.95 \beta_{1}$ & 0.83003 & 1.12073 & 1.12070 & 0.72289 \\
\hline$\beta_{1}$ & 0.91562 & 1.28374 & 1.28374 & 0.92021 \\
\hline$+0.95 \beta_{1}$ & 1.00121 & 1.44676 & 1.44680 & 1.11753 \\
\hline
\end{tabular}

Table (7): 95\% Confidence intervals on intercept and slope, standard grid.

\begin{tabular}{|c|c|c|c|}
\hline Response & Correlation Coeff., $\sigma$ & RMS Error, Sy.x & Comment \\
\hline PFAIP & 0.98573 & 0.00479 & Not Biased \\
\hline DPH/P & 0.74131 & 0.00915 & Biased \\
\hline DPT/P & 0.93118 & 0.00922 & Biased \\
\hline DPC/P & 0.97414 & 0.00151 & Not Biased \\
\hline
\end{tabular}

Table (8): Systematic (biasing) errors over operability space, standard grid.

\begin{tabular}{|c|c|c|c|}
\hline Response & $\begin{array}{c}\text { HSCT Nominal } \\
\text { Goal }\end{array}$ & $\begin{array}{c}\text { HSCT Maneuver } \\
\text { Goal }\end{array}$ & $\begin{array}{c}0.95 \% \text { Confidence } \\
\text { Interval }^{*}\end{array}$ \\
\hline PFAIP & N/A & N/A & \pm 0.01072 \\
\hline DPH/P & 0.03 & 0.05 & \pm 0.01011 \\
\hline DPT/P & 0.03 & 0.05 & \pm 0.02051 \\
\hline DPC/P & 0.06 & 0.08 & \pm 0.02066 \\
\hline
\end{tabular}

Table (9): Random (precision) errors over operability space, standard grid.

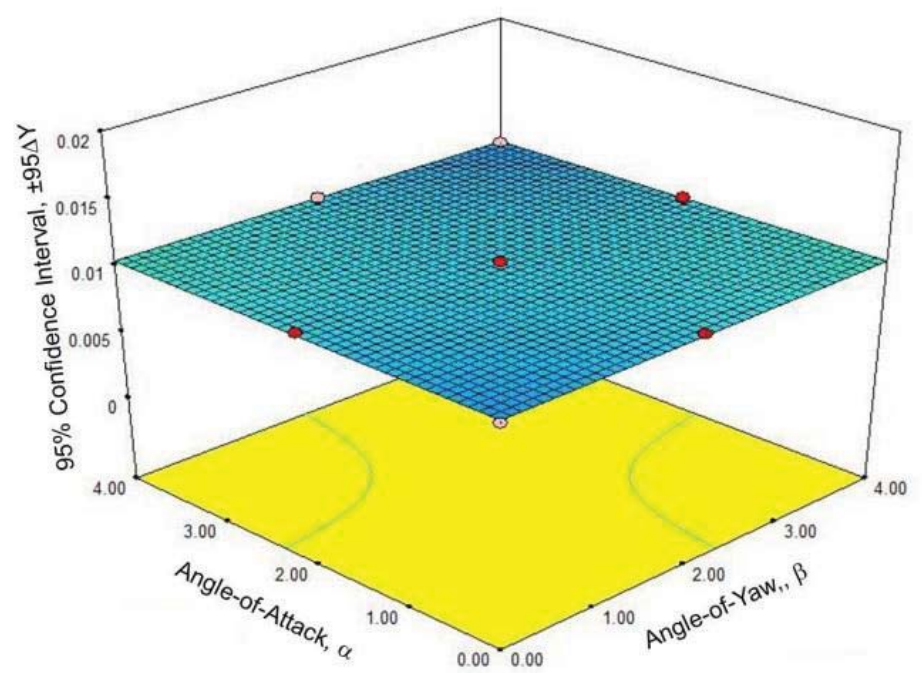

(a) PFAIP $\alpha * \beta$ interaction.

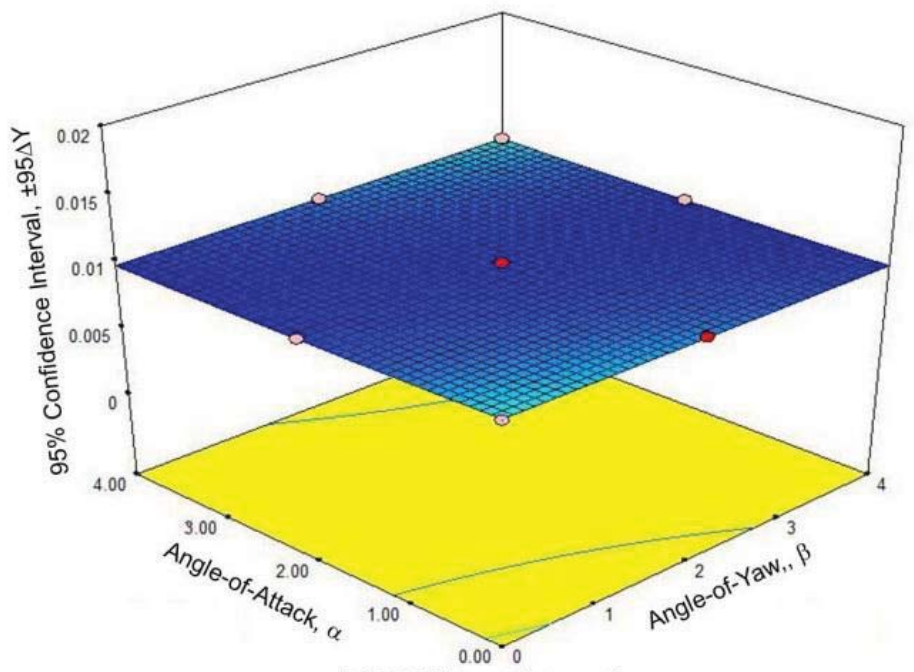

(b) $\mathrm{DPH} / \mathrm{P} \alpha * \beta$ interaction.

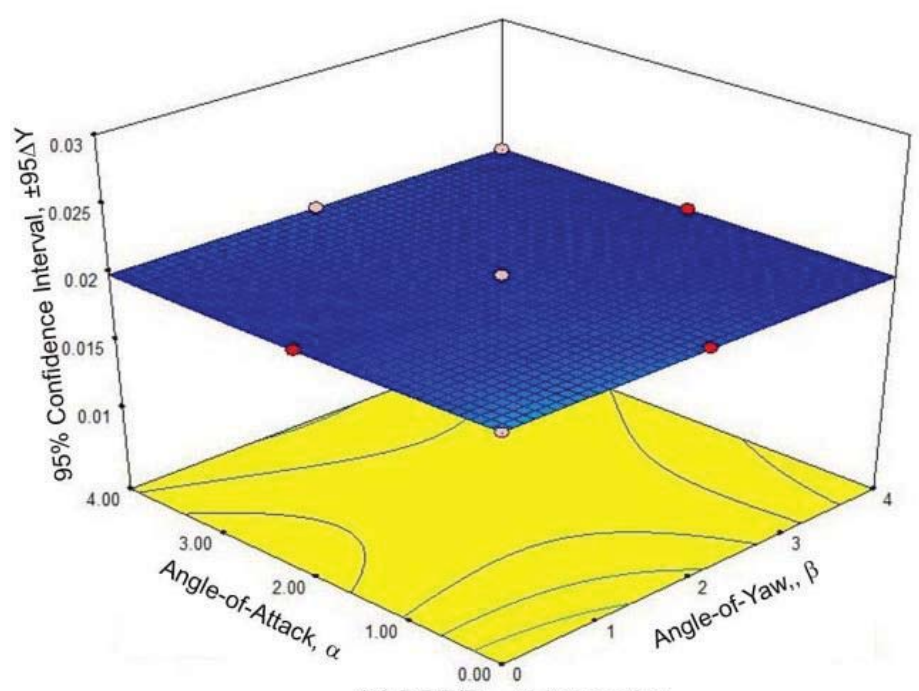

(c) DPT/P $\alpha * \beta$ interaction.

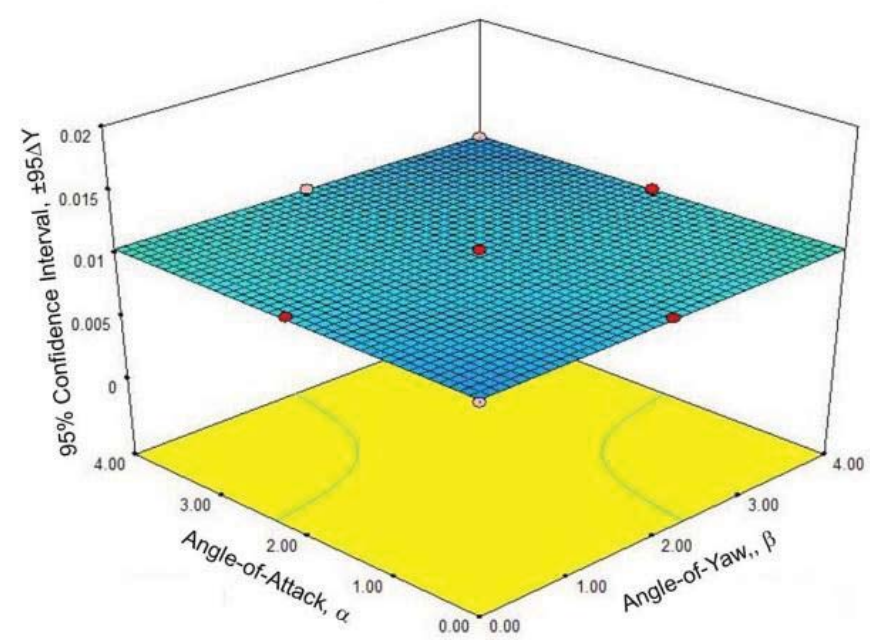

(d) DPC/P $\alpha * \beta$ interaction.

Figure (10): Response surface $\pm 95 \%$ confidence interval, $M_{0}=1.70$. 